\title{
Identification of differentially expressed profiles of IncRNAs and mRNAs in ER-negative and HER-2 positive breast cancer
}

\author{
Cheng Qian, Ming Guan, Cheng Si, Haipeng Shen, Taobo Jin, Tianya Zhang
}

Department of Breast and Thyroid Surgery, Zhuji People's Hospital of Zhejiang Province, Zhejiang, China

Submitted: 14 March 2017

Accepted: 18 May 2017

Arch Med Sci Civil Dis 2017; 2: e148-e160

DOI: https://doi.org/10.5114/amscd.2017.71413

Copyright $@ 2017$ Termedia \& Banach

\section{Abstract}

Introduction: Breast cancer is one of the most common malignant tumors in the United States. However, the molecular mechanism involved in the progression of breast cancer has remained unclear. Long non-coding RNAs (IncRNA) have been reported as key regulators in the progression and metastasis of cancer.

Material and methods: In this study, we identified significantly differentially expressed mRNAs and IncRNAs in breast cancer using the GSE70947 dataset. Gene ontology (GO) and KEGG pathway was used to explore the key roles of differentially expressed IncRNAs in breast cancer. The dysregulated IncRNAs and mRNAs expression profiles in HER2-positive and ER-negative breast cancer were also analyzed in this study.

Results: Our results showed that PVT1, LOC145837, FLJ40504 and FLJ45983 were significantly decreased in HER2-positive and ER-negative breast cancers. We also constructed the PVT1, LOC145837, FLJ40504 and FLJ45983 mediated CRNA networks in HER2-positive and ER-negative breast cancers. Moreover, using the Betastasis dataset, we found that high PVT1 expression levels were associated with a lower survival rate in breast cancer patients.

Conclusions: These results elucidate the functions of IncRNAs and provide useful information for exploring therapeutic candidate targets and new molecular biomarkers for ER-negative and HER-2 enriched subtype breast cancer.

Key words: long non-coding RNA, ER-negative, ceRNA networks, HER-2 positive, breast cancer.

\section{Introduction}

Breast cancer $(\mathrm{BC})$ is one of the most common malignant tumors, with more than 200,000 newly diagnosed cases in the United States per year $[1,2]$. According to Prat et al., the estrogen receptor (ER) was overexpressed in nearly $70 \%$ of breast cancer cases [3]. Of note, the molecular subtypes of ER are the main indicator of anti-hormonal therapy for breast cancer [4]. Moreover, ER-negative (ER-) BC exhibited dismal survival rates due to the highly aggressive and metastatic behavior [5, 6]. HER2, also known as HER2/neu and ERBB2, was found to be overexpressed in about $20-30 \%$ of early-stage breast cancer cases [7]. A wealth of evidence has shown that HER-2-positive breast cancer is associated

\author{
Corresponding author: \\ Dr. Cheng Qian \\ Department of Breast \\ and Thyroid Surgery \\ Zhuji People's Hospital \\ of Zhejiang Province \\ 311800 Zhuji \\ Zhejiang, China \\ Phone: +86 13585896239 \\ E-mail: chengqian2010@ \\ qq.com
}


with a more aggressive phenotype and shorter survival time [8]. However, how HER2 and ER dysregulation in breast cancer effect the progression of breast cancer remains unclear [9].

Previous reports had shown that non-coding RNAs (ncRNAs) acted as key regulators in the progression and metastasis of cancer [10, 11]. The best characterized ncRNAs is microRNAs (miRNAs). miRNAs are a class of small ncRNAs and mediate the post-transcriptional regulation of gene expression [12]. Recently, more and more researchers have paid attention to the long noncoding RNAs (IncRNAs). Increasingly reports have shown that IncRNAs can regulate expression of protein-coding genes through transcriptional, post-transcriptional and post-translational regulation [13].

Lately, several reports have also shown that altered expression of IncRNAs also played important roles in regulating breast cancer progression. MALAT1 was up-regulated in multiple human malignancies. In breast cancer, MALAT1 interacted with the estrogen receptor and predicted poor survival. Lin et al. also reported that LINK-A IncRNA activates HIF1 $\alpha$ signaling to promote breast cancer glycolysis reprogramming and tumorigenesis in triple-negative breast cancer [14]. In 2016, Niknafs et al. identified a cohort of breast cancer-associated and estrogen-regulated IncRNAs and demonstrate that DSCAM-AS1 mediates tumorous progression and tamoxifen resistance [15]. However, few studies have focused on the dysregulation of IncRNAs in the HER-2-enriched and ER-negative subtype breast cancer.

In this study, we aimed to identify differentially expressed IncRNAs and mRNAs in breast cancer by analyzing previously published datasets. To understand the biological roles of these differently expressed genes, we performed GO and KEGG analysis to explore the potential roles of dysregulating IncRNAs. We also conducted a bioinformatic analysis to identify the IncRNA-miRNA-mRNA regulatory axis in breast cancer. We hope that our work will be useful to identify candidate therapeutic targets and new molecular biomarkers for HER-2enriched and ER-negative subtype breast cancer.

\section{Material and methods}

\section{Microarray data and data preprocessing}

Microarray data were downloaded from the study by Quigley DA, which was referenced in the Gene Expression Omnibus (GEO) database (www. ncbi.nlm.nih.gov/geo/) under accession number GSE70947. In this dataset, expression profiles were obtained from 81 biopsies obtained from 41 patients diagnosed with follicular lymphoma by using Affymetrix HG U133 Plus 2.0 Gene Chip.
mRNAs having fold changes $\geq 2$ and $p$-values $<0.05$ were selected as of significantly differential expression.

\section{LncRNA classification pipeline}

To evaluate the IncRNA expressions in microarray data, we applied a pipeline described by Zhang et al. [16] to identify the probe sets uniquely mapped to IncRNAs from the Affymetrix array by using the following criteria. For the probe sets with Refseq IDs, we retained those labeled as "NR_" (NR indicates non-coding RNA in the Refseq database). For the probe sets with Ensembl gene IDs, we retained those annotated with "IncRNA", "processed transcripts", "non-coding" or "misc RNA" in Ensembl annotations. Then, we filtered the probe sets obtained from last step by filtering out pseudogenes, rRNAs, microRNAs, tRNAs, snRNAs and snoRNAs. Finally, we obtained 2448 annotated IncRNA transcripts with corresponding Affymetrix probe IDs.

LncRNAs having fold changes $\geq 2$ and $p$-values $<0.05$ were selected as of significantly differential expression.

\section{Functional group analysis}

GO analysis and KEGG analysis were applied to determine the biological roles of these differentially expressed mRNAs, based on the freely available online MAS system provided by the CapitalBio company (Molecule Annotation System, http://bioinfo.capitalbio.com/mas3/). The $p$-value (hypergeometric $p$-value) denoted the significance of the pathway correlated to the conditions. The recommend $p$-value cut-off is 0.05 .

\section{Hierarchical clustering analysis}

To generate an overview of IncRNA and mRNA expression profiles between the $F L$ and DLBCL, hierarchical clustering analysis was performed based on the expression value.

\section{PPI network and module analysis}

The interaction relationships of the proteins encoded by DEGs were searched by STRING online software [17], and the combined score $>0.4$ was used as the cut-off criterion. The PPI network was visualized using Cytoscape software [18]. Then, modules of the PPI network were screened by CFinder software [19], and the parameter $k$ was set to 6 .

\section{Statistical analysis}

The numerical data were presented as mean \pm standard deviation (SD) of at least three determinations. Statistical comparisons between groups 
of normalized data were performed using the $t$-test or Mann-Whitney $U$-test according to the test condition. Values of $p<0.05$ were considered statistically significant with a $95 \%$ confidence level.

\section{Results}

\section{Comprehensive analysis of significant differential expression of mRNAs and IncRNAs in breast cancer}

To identify significant differential expression of mRNAs and IncRNAs in breast cancer, we analyzed a publicly available gene expression dataset, GSE70947. The GSE70947 database includes 148 paired breast cancer samples. Genes with FC $>2$ for both up- or down-regulation and a $p$-value $<0.05$ were identified as significantly differentially expressed. Compared to the normal breast tissues, a total of 1,382 IncRNAs displayed differential expression in tumor tissues, including 23 upregulated IncRNAs and 57 downregulated IncRNAs (Figure $1 \mathrm{~B}$ ). We found 4248 differently expressed mRNAs, of which 2094 were upregulated and 2153 were downregulated (Figure 1 A). Hierarchical clustering showed systematic variations in the expression of IncRNAs and mRNAs in the breast cancer samples.

\section{GO and KEGG analysis of differentially expressed mRNAs}

To determine the potential roles of differentially expressed mRNAs, we performed $\mathrm{GO}$ and KEGG pathway analysis using mas3.0. GO analysis showed that the dysregulated genes were mainly involved in regulating signal transduction, development, regulation of transcription, oxidation reduction, cell adhesion and the cell cycle (Figure $1 \mathrm{C}$ ). Meanwhile, KEGG pathway analysis revealed that dysregulated genes were primarily enriched in pathways associated with the MAPK signaling pathway, insulin signaling pathway, and Jak-STAT signaling pathway (Figure $1 \mathrm{D}$ ).

\section{GO and KEGG analysis of differentially expressed IncRNAs}

To predict the functions of the differentially expressed IncRNAs, we adopted methods as described by Guttman and Rinn [20] and Shen et al. [21]. We first constructed co-expression networks to identify the correlation between differentially expressed mRNAs and IncRNAs by using GSE70947. Next, we performed GO and KEGG pathway analysis for each given IncRNA by using the set of co-expressed mRNAs. In this study, the top 50 related mRNAs of each IncRNA were classified according to GO terms. According to the KEGG pathway analysis, dysregulated IncRNAs were primarily enriched in pathways associated with the insulin signaling pathway, MAPK signaling pathway, PPAR signaling pathway and adipocytokine signaling pathway (Figure $1 \mathrm{~F}$ ). GO analysis revealed that the dysregulated IncRNAs were enriched in signal transduction, cell adhesion, cell cycle, mitosis, and cell division (Figure $1 \mathrm{E}$ ).

\section{Identification of key mRNAs and IncRNAs involved in ER-negative breast cancer}

To investigate differences in the expression of mRNAs and IncRNAs between ER-negative and ER-positive breast cancer, we analyzed the GSE70947 database. We found that 162 mRNA transcripts were up-regulated and 812 mRNA transcripts were down-regulated in ER-negative samples compared to the ER-positive samples (Figure 2 A). Of note, we identified 10 dysregulated InCRNAs in ER-negative breast cancer. C21orf81, PVT1, NBPF22P, PP14571, CYP4Z2P, FLJ40504, LOC440335, LOC145837, and FLJ45983 were down-regulated and LOC375196 was up-regulated in ER-negative samples (Figure 2B).

\section{Functional analysis of IncRNA-associated}

\section{PPI modules in ER-negative breast cancer}

We identified a series of dysregulated IncRNAs, including C21orf81, PVT1, NBPF22P, PP14571, CYP4Z2P, FLJ40504, LOC440335, LOC145837, FLJ45983 and LOC375196, in ER-negative breast cancer. Next, we analyzed co-expressed mRNAs of these IncRNAs and examined whether the mRNAs were connected by PPIs.

Based on the information in the STRING database, we first constructed a protein-protein interaction network in the ER-negative breast cancer. The PPI network of the DEGs had 8 nodes comprising ERBB3, KRT8, KRT19, MYB, GATA3, $X B P 1$, FOXA1 and ESR1 (Figure 2 C). Among these genes, ESR1 showed the highest node degree, which was 9.

By analyzing co-expressed mRNAs, we found that these IncRNAs were associated with regulation of transcription, response to estrogen stimulus, tumor necrosis factor-mediated signaling pathway, negative regulation of cell growth, negative regulation of cell adhesion, interspecies interaction between organisms and cell-cell signaling (Figure $3 \mathrm{~A}$ ).

\section{Identification of key mRNAs and IncRNAs involved in HER2-positive breast cancer}

In this study, we also identified differentially expressed mRNAs and IncRNAs between HER2-negative and HER2-positive breast cancer. A total of 373 mRNA transcripts were found to be down-regulated and 137 mRNA transcripts were 
A

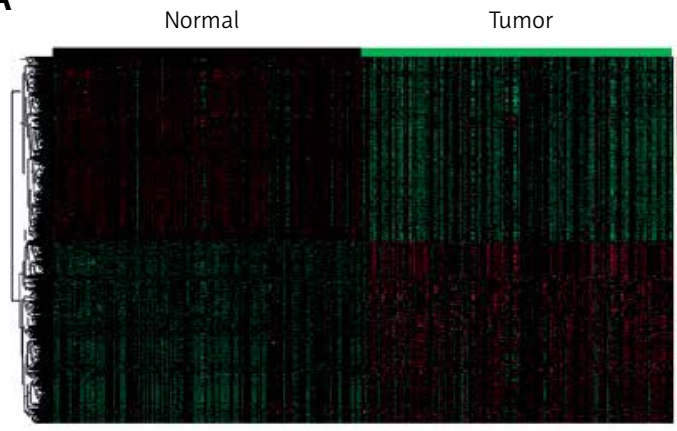

C Differentially expressed mRNAs related biological processes

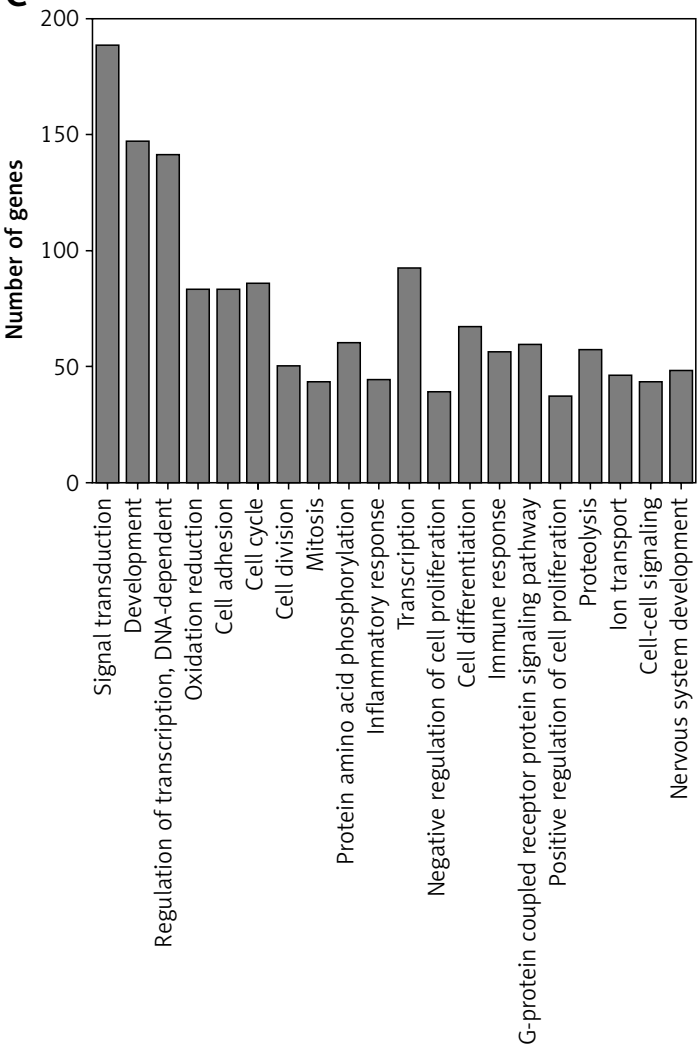

B

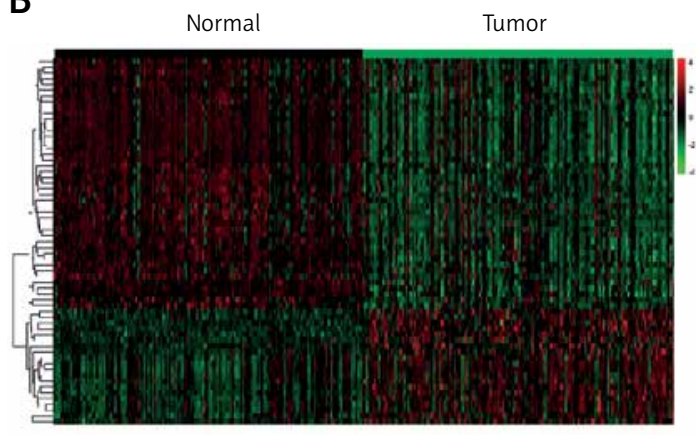

D

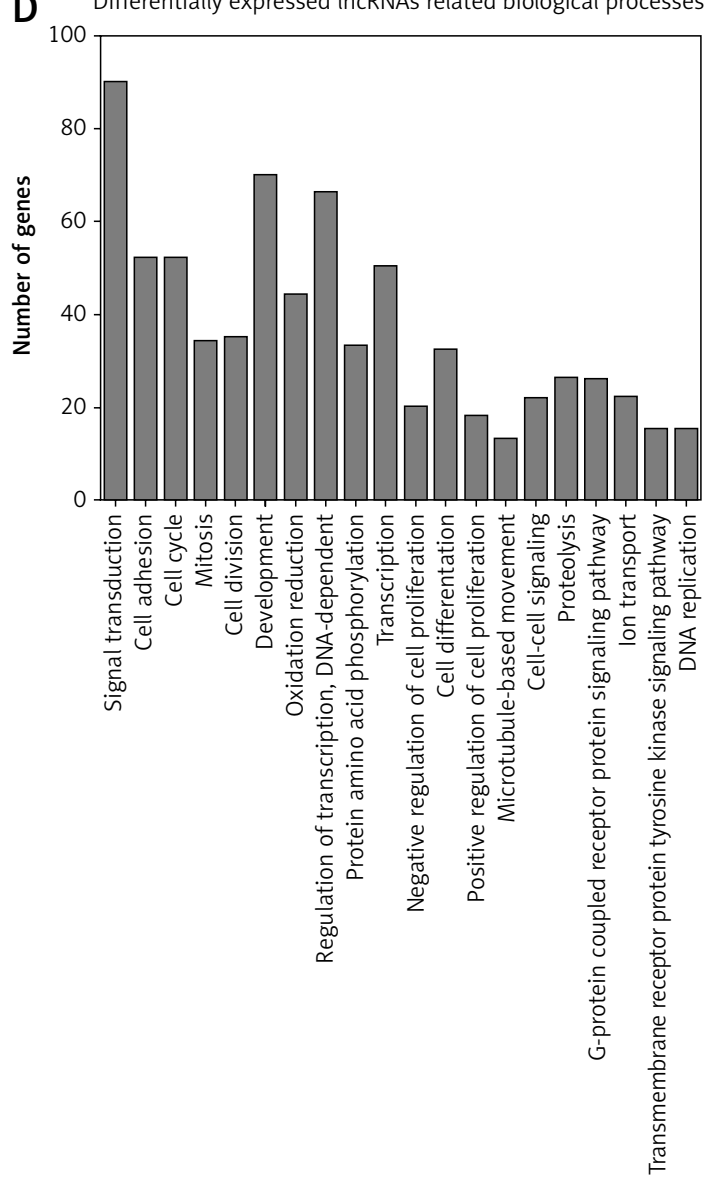

$\mathrm{E}$

Differentially expressed mRNAs related pathways Hedgehog signaling pathway

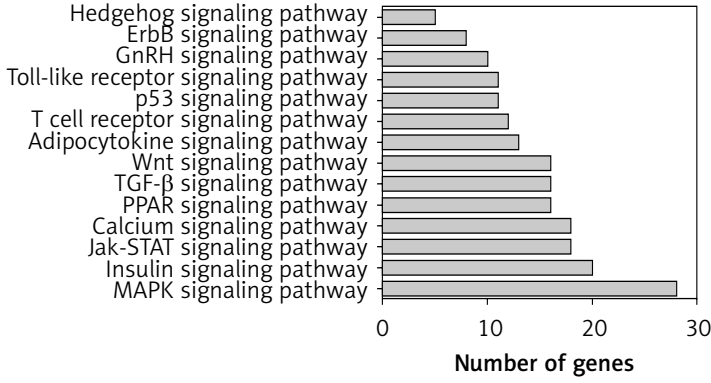

F Differentially expressed IncRNAs related pathways Toll-like receptor signaling pathway TGF- $\beta$ signaling pathway ErbB signaling pathway p53 signaling pathway Jak-STAT signaling pathway Calcium signaling pathway Wnt signaling pathway Adipocytokine signaling pathway PPAR signaling pathway
MAPK signaling pathway Insulin signaling pathway

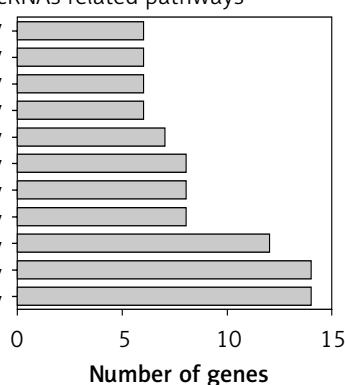

Figure 1. GO and KEGG analysis of differentially expressed mRNAs and IncRNAs in breast cancer. Differentially expressed mRNAs (A) and IncRNAs (B) in breast cancer patient tumors versus adjacent matched normal tissue obtained from GSE70947 are represented by heatmaps. GO (C) and KEGG (D) pathway analysis of the dysregulated mRNAs. GO (E) and KEGG (F) analysis of the dysregulated IncRNAs 
A

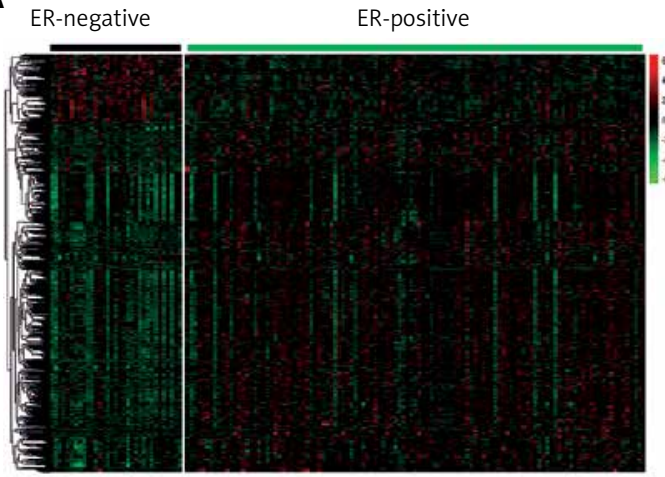

ER-negative

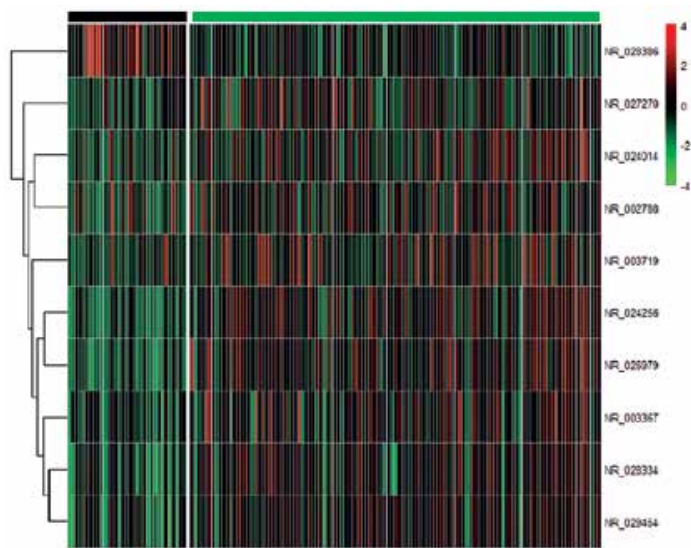

C

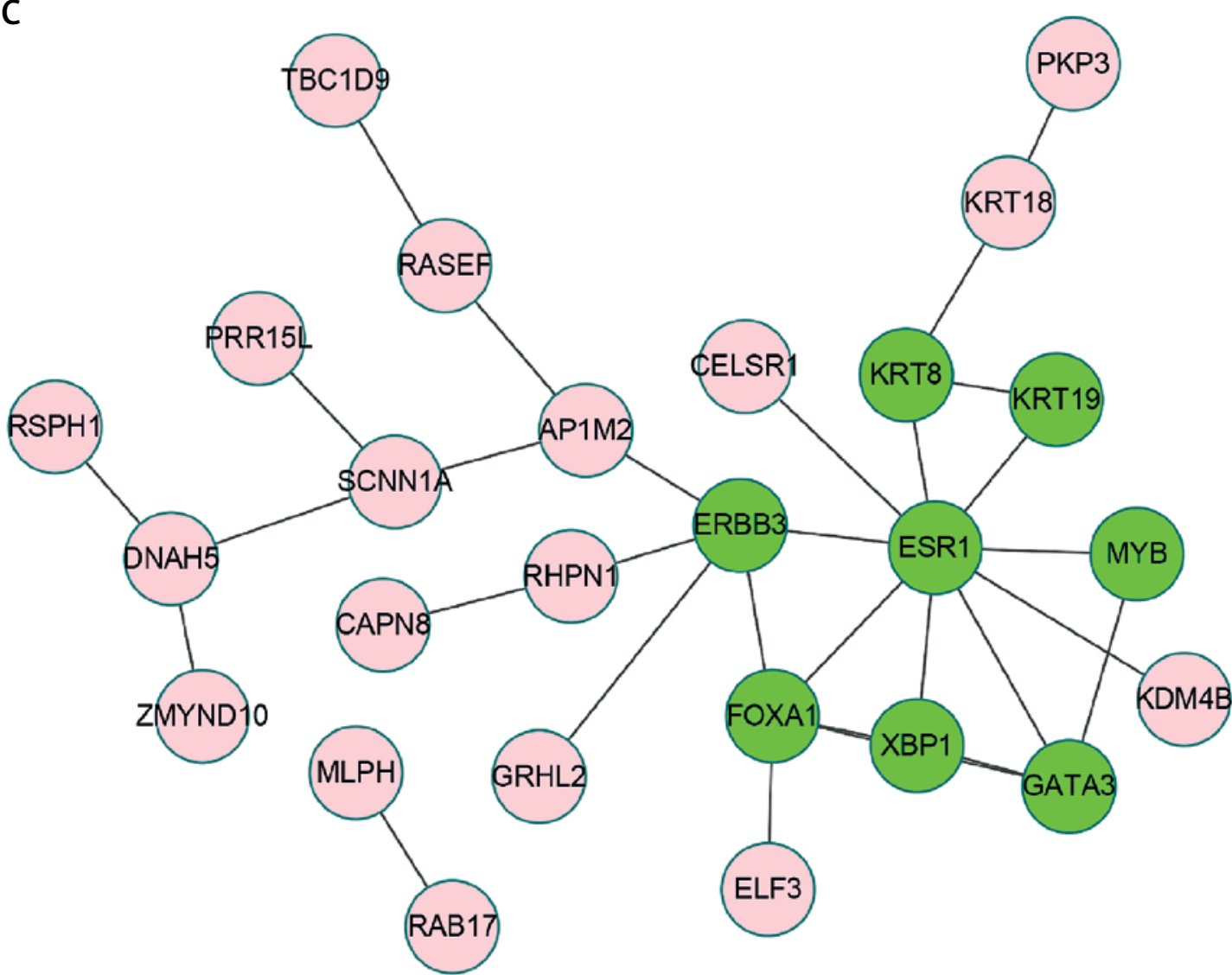

Figure 2. Functional analysis of IncRNA-associated PPI modules in ER-negative breast cancer. Identification of key mRNAs (A) and IncRNAs (B) in ER-negative breast cancer. C - PPI network for the proteins encoded by the DEGs in ER-negative breast cancer

found to be induced in HER2-positive samples compared to the HER2-negative samples (Figure 4 A). Ten IncRNAs - FLJ45983, LOC440335, NEURL3, C9orf122, FLJ40504, LOC145837, PVT1, NCRNA00173, EGOT, and LOC283867 - were down-regulated in HER2-positive samples. Interestingly, we did not find down-regulated IncRNAs in HER2-positive samples (Figure 4 B).
Functional analysis of IncRNA-associated PPI modules in HER2-positive breast cancer

The PPI network of the DEGs in HER2-positive breast cancer had 17 nodes including ERBB3, MUC1, FOXA1, CDH1, MMP3, GATA3 and MYB. FLJ45983, LOC440335, NEURL3, C9orf122, FLJ40504, LOC145837, PVT1, NCRNA00173, EGOT, and LOC283867 were found to be down-regulated in 


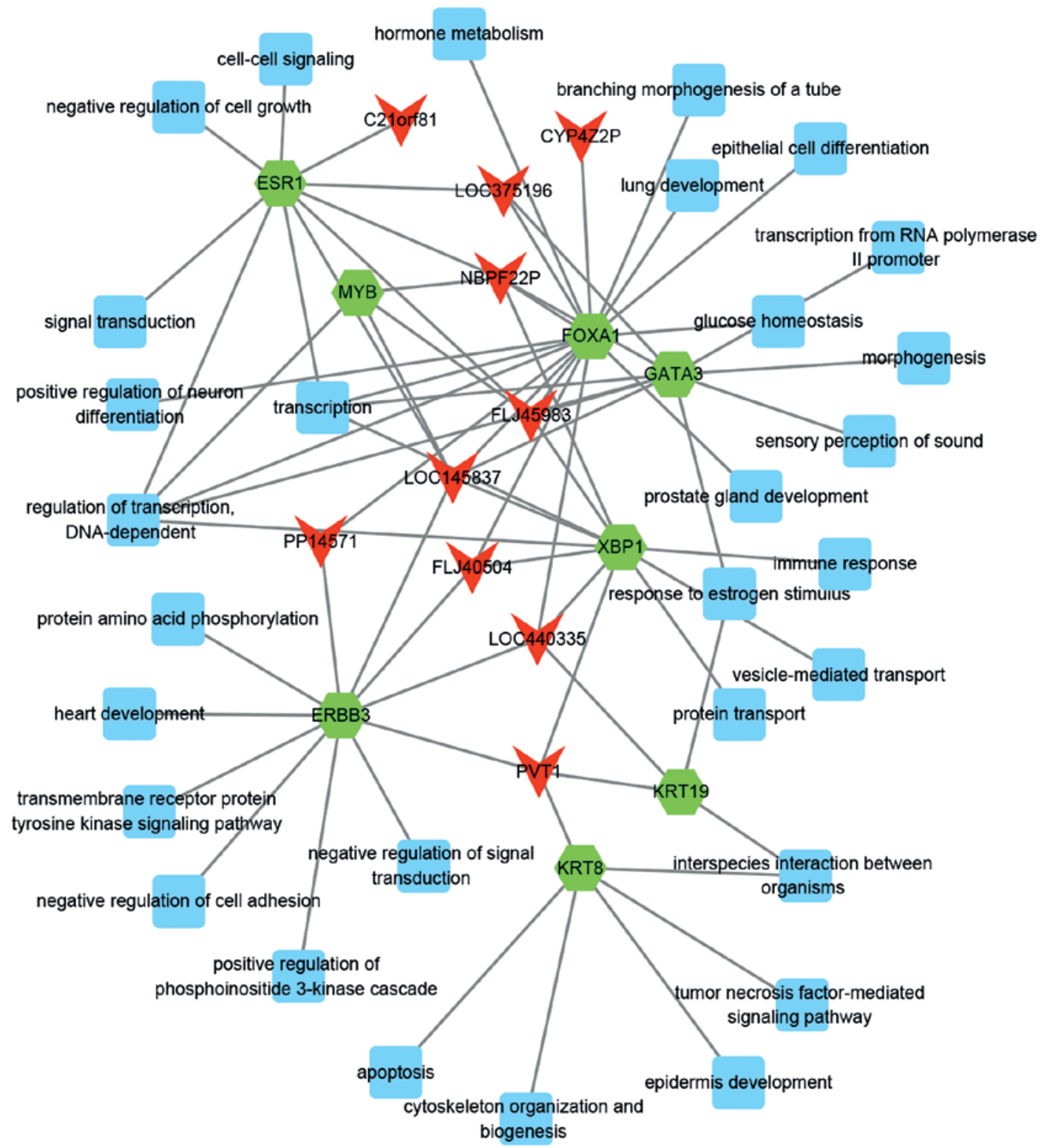

Figure 3. Functional analysis of IncRNA-associated PPI modules in ER-negative breast cancer

HER2-positive breast cancer (Figure 4C). By analyzing co-expressed mRNAs of each IncRNA, we found that these IncRNAs were associated with collagen catabolism, the estrogen receptor signaling pathway, glucose homeostasis, hormone metabolism, negative regulation of cell adhesion, positive regulation of neuron differentiation, the response to estrogen stimulus and regulation of cell proliferation (Figure 5).

\section{Construction of the PVT1, LOC145837,} FLJ40504 and FLJ45983 mediated ceRNA networks in HER2-positive and ER-negative breast cancers

In this study, analysis of the GSE70947 database showed that PVT1, LOC145837, FLJ40504 and FLJ45983 were significantly decreased in HER2-positive (Figure 6 A) and ER-negative breast cancers (Figure 7 A). To explore the molecular mechanisms involved in these IncRNAs regulating breast cancer progression, we constructed IncRNA-miRNA-mRNA ceRNA networks based on our analysis.

First, we constructed a co-expression network based on correlation analysis between the differentially expressed IncRNAs and mRNAs. The IncRNA-mRNA interaction was integrated into the co-expression networks according to the positive regulation and only gene pairs with $|R|>0.7$ were selected. We next predicted the interactions between differentially expressed IncRNAs and their target miRNAs theoretically by using miRcode 
A

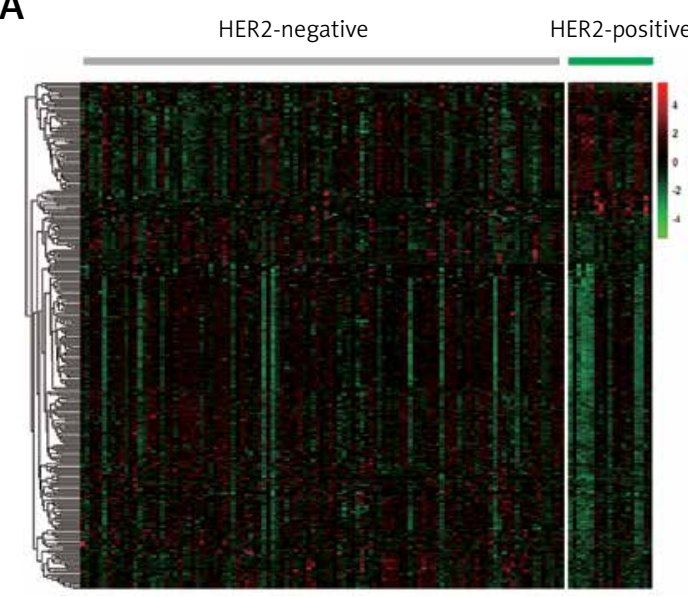

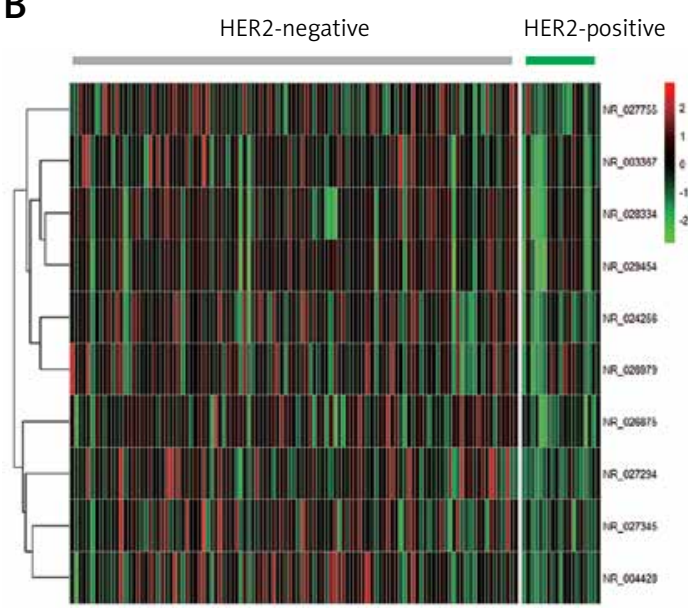

C

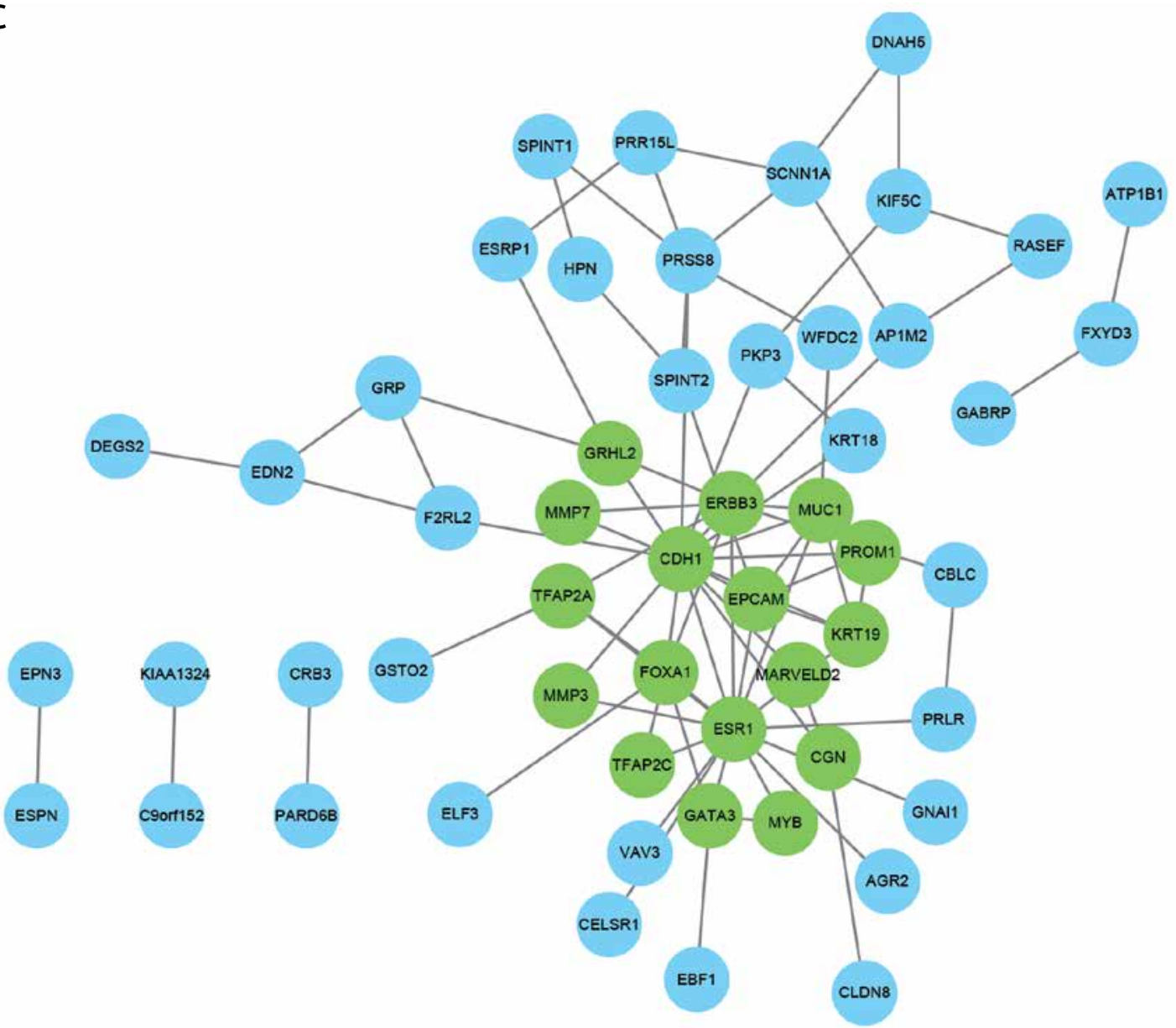

Figure 4. Functional analysis of IncRNA-associated PPI modules in HER2-positive breast cancer. Identification of key mRNAs (A) and IncRNAs (B) in HER2-positive breast cancer. C - PPI network for the proteins encoded by the DEGs in HER2-positive breast cancer

[21]. Finally, TargetScan [22] and StarBase databases were both used to identify miRNAs which suppress mRNAs. The networks were drawn using Cytoscape 3.0.

The ceRNA network in the ER-negative breast cancer tumor group comprised 4 IncRNAs, 32
miRNAs, and 29 mRNAs (Figure 7 B). The ceRNA network in the HER2-positive breast cancer tumor group comprised 4 IncRNAs, 28 miRNAs, and 27 mRNAs (Figure $6 \mathrm{~B}$ ). Our results showed that PVT1 played the most important roles. The networks were drawn using Cytoscape 3.0. 


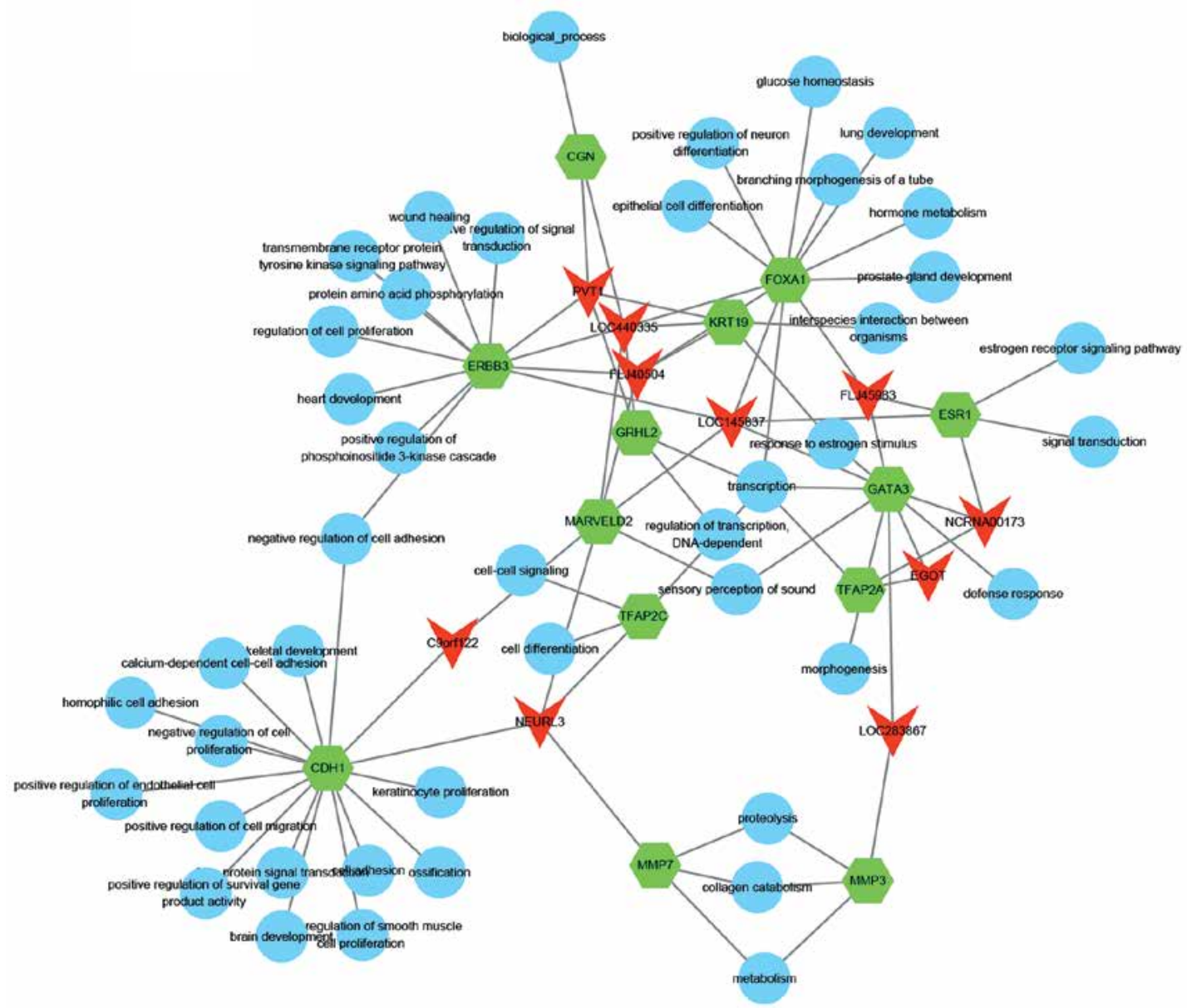

Figure 5. Functional analysis of IncRNA-associated PPI modules in HER2-positive breast cancer

\section{Alterations of IncRNAs expression and prognosis in breast cancer}

To evaluate the possible prognostic value of PVT1, LOC145837, FLJ40504 and FLJ45983, we analyzed the other dataset which was downloaded from Betastasis (http://www.betastasis. com/). Expression levels of three IncRNA (PVT1, LOC145837, FLJ40504) with survival data were included in this dataset. As shown in Figures $8 \mathrm{~A}-\mathrm{C}$, Kaplan-Meier analysis showed that patients with high PVT1 expression levels had decreased overall survival compared to those with low PVT1 levels $(p=0.045)$. However, we found that the expression levels of LOC145837 and FLJ40504 were not associated with survival status of breast cancer patients.

We also combined PVT1 expression and ER status to stratify the possibility of breast cancer survival. We found that high PVT1 expression levels were also associated with a lower survival rate in both ER-negative and ER-positive patients (Figures $8 \mathrm{D}-\mathrm{E})$. These results suggested that PVT1 may act as an oncogene in breast cancer, which was consistent with previous reports [23].

\section{Discussion}

Breast cancer is one of the most common malignant tumors in the United States [24]. However, the molecular mechanism involved in the progression of breast cancer has remained unclear. Recently studies have shown that IncRNAs play key roles in tumorigenesis, cancer progression, and metastasis [1]. Therefore, it was critically important to investigate the potential roles of IncRNAs in breast cancer. In the present study, we analyzed the GSE70947 database and identified 23 upregulated LncRNAs and 57 downregulated LncRNAs in breast cancer compared to normal breast tissues. We also found 4248 differently expressed mRNAs, of which 2094 were upregulated and 2153 were downregulated.

Recently, several reports have shown that some IncRNAs play important roles in regulating breast cancer progression. For example, MALAT1 interacted with the estrogen receptor and was associated with a poor survival rate [22]. Lin et al. also reported that LINK-A IncRNA activates HIF1 $\alpha$ signaling to promote breast cancer glycolysis reprogramming and tumorigenesis in triple-negative breast cancer $[2,25]$. In 2016, Niknafs et al. 
A
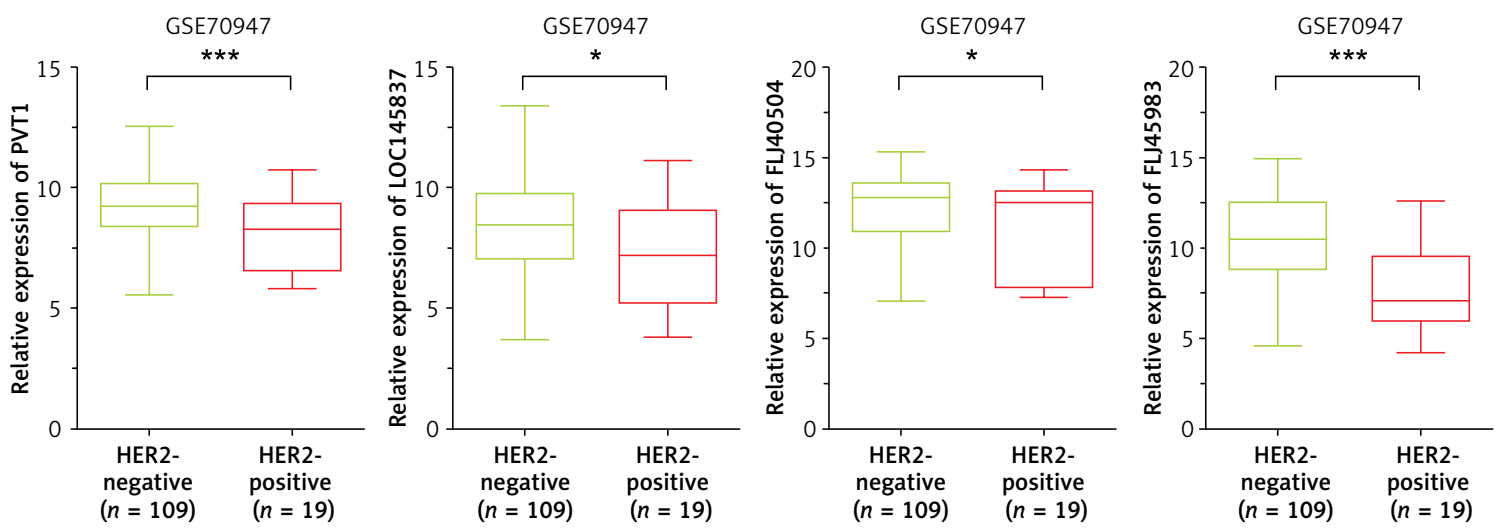

B

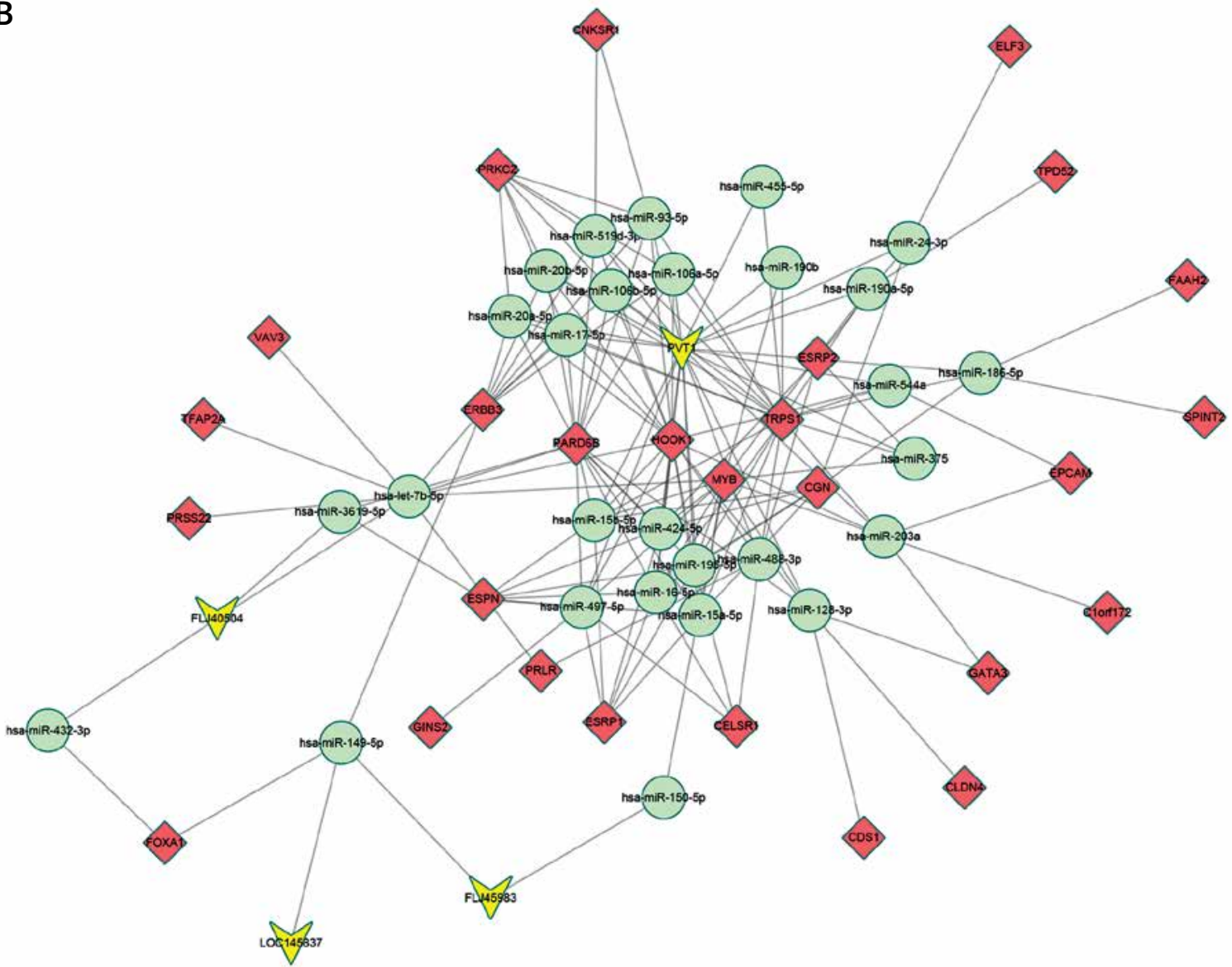

Figure 6. Construction of the PVT1, LOC145837, FLJ40504 and FLJ45983 mediated ceRNA networks in ER-negative breast cancers. A - PVT1, LOC145837, FLJ40504 and FLJ45983 expression levels in ER-negative breast cancers compared to ER-positive breast cancers in publicly available gene expression data GSE70947. B - PVT1, LOC145837, FLJ40504 and FLJ45983 mediated ceRNA networks in ER-negative breast cancers

identified a cohort of breast cancer-associated and estrogen-regulated IncRNAs and demonstrated that DSCAM-AS1 mediates tumor progression and tamoxifen resistance [15]. In this study, to predict the functions of the differentially expressed IncRNAs, we first constructed co-expression networks and we performed GO and KEGG analysis for each given IncRNA by using the set of co-expressed mRNAs. According to the KEGG pathway analysis, dysregulated IncRNAs were primarily enriched in pathways associated with the insulin signaling pathway, MAPK signaling pathway, PPAR signaling pathway and adipocytokine signaling pathway. $\mathrm{GO}$ analysis revealed that the 
A
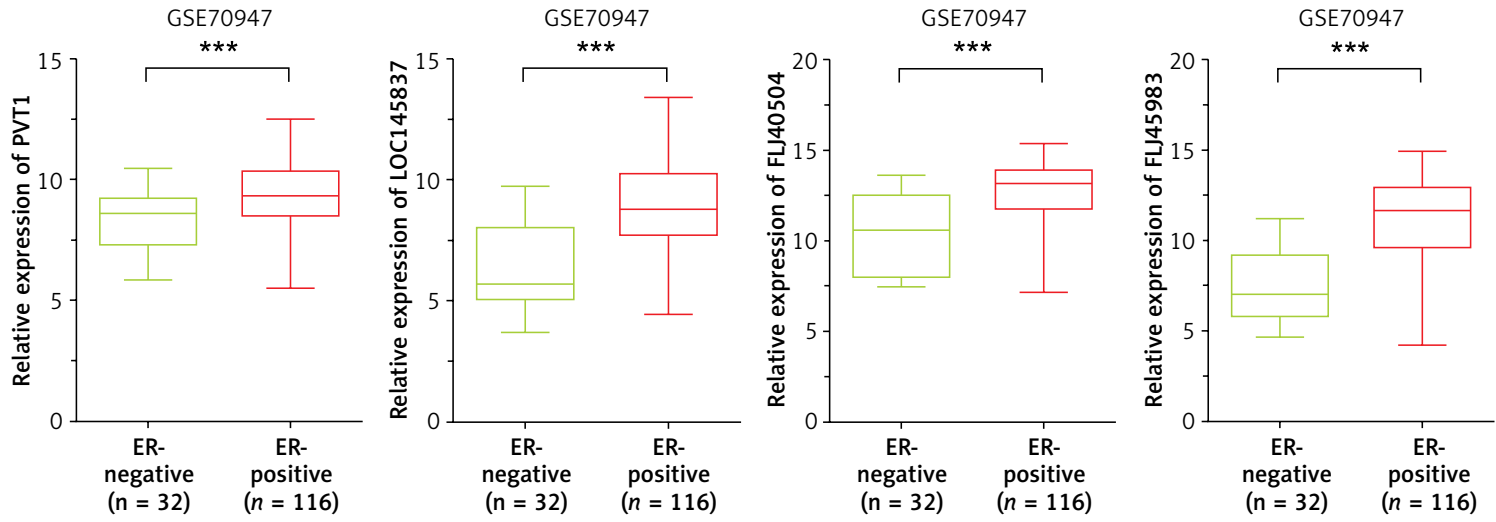

\section{B}

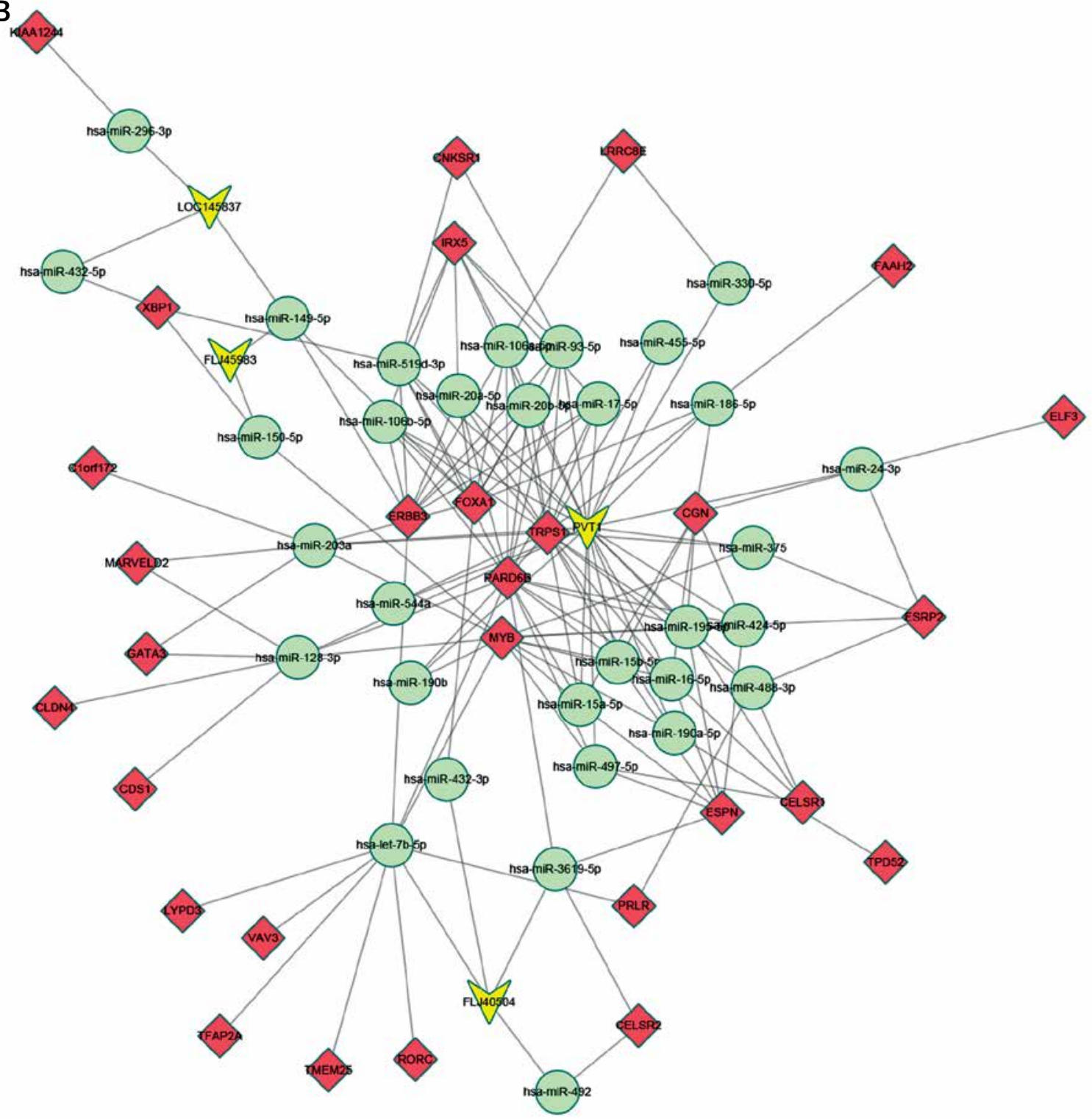

Figure 7. Construction of the PVT1, LOC145837, FLJ40504 and FLJ45983 mediated ceRNA networks in HER2-positive breast cancers. A - PVT1, LOC145837, FLJ40504 and FLJ45983 expression levels in HER2-positive breast cancers compared to HER2-negative breast cancers in publicly available gene expression dataset GSE70947. B - PVT1, LOC145837, FLJ40504 and FLJ45983 mediated ceRNA networks in HER2-positive breast cancers 
A

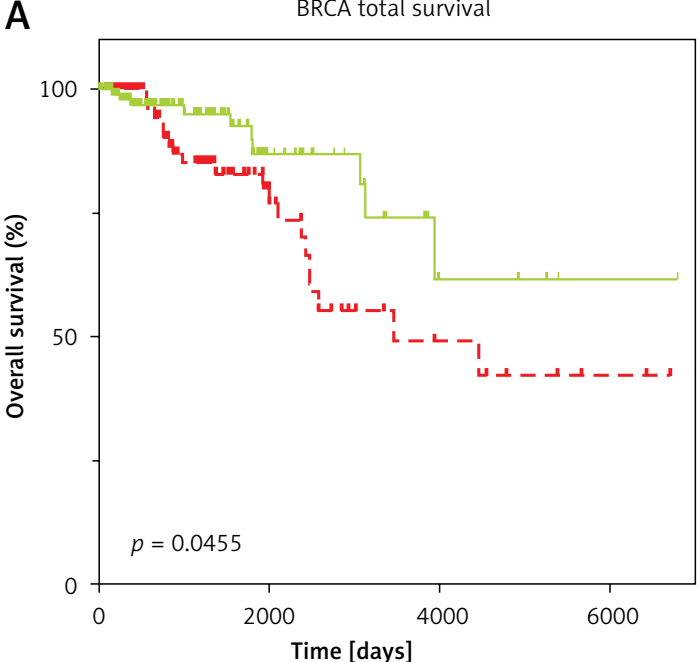

- ᄂ - PVT1-high $(n=113) \longrightarrow$ PVT1-low $(n=113)$

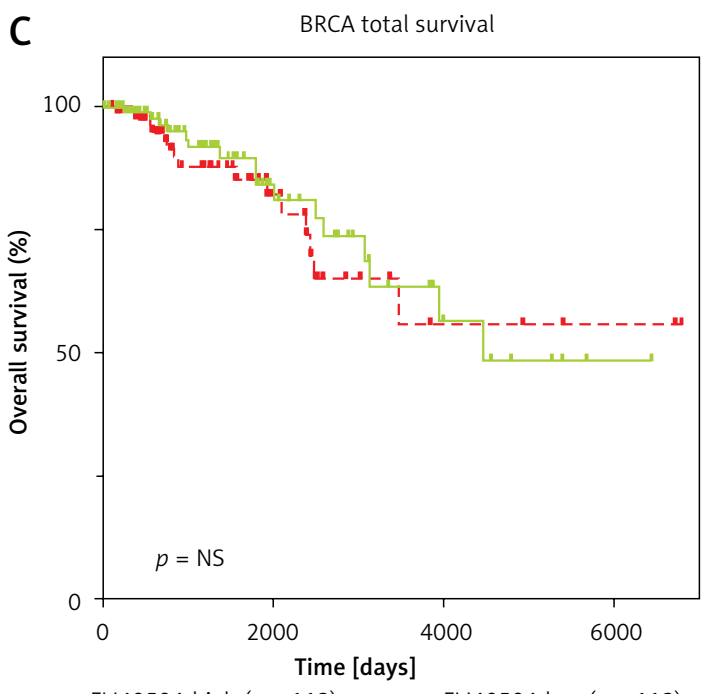

- $\perp$ - FLJ40504-high $(n=113)$

FLJ40504-low $(n=113)$

$E$

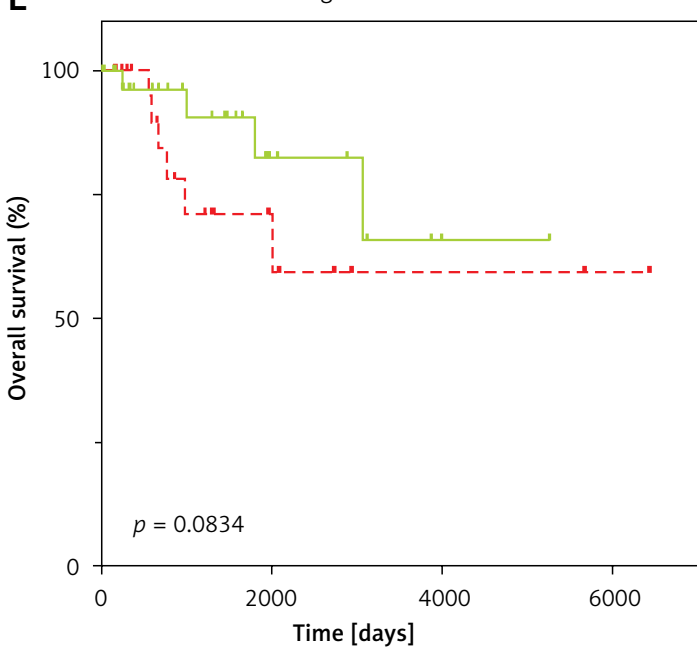

- ᄂ - PVT1-high $(n=26) \longrightarrow$ PVT1-low $(n=33)$
B

BRCA total survival

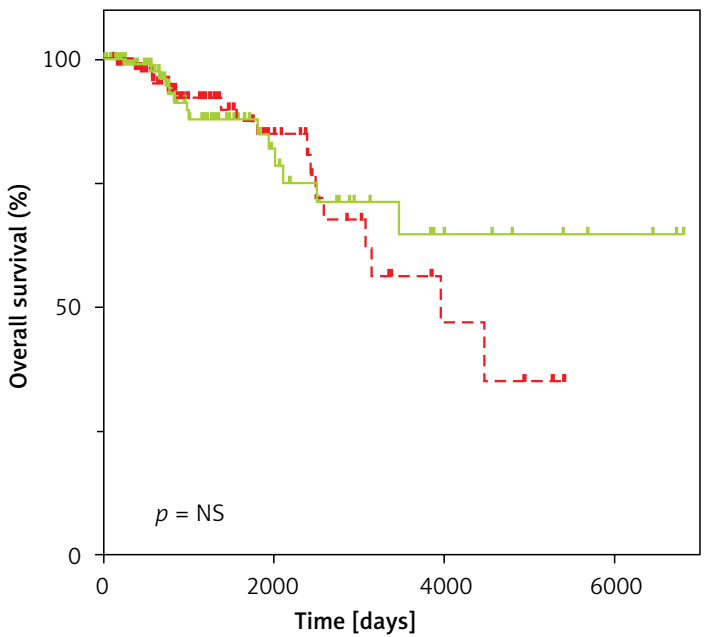

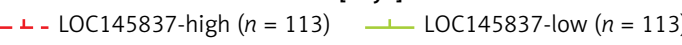

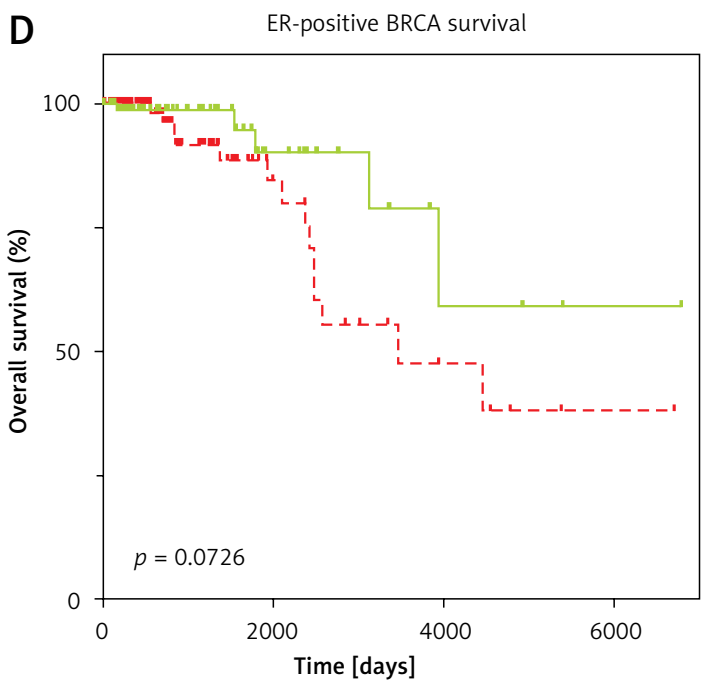

— $\perp$ - PVT1-high $(n=85) \_$PVT1-low $(n=77)$

Figure 8. Kaplan-Meier analysis of overall BRCA survival rate. Relationship between overall survival rate and expression level of PVT1 (A), LOC145837 (B) and FLJ40504 (C). Relationship between overall survival rates of ER-positive BRCA (D) and ER-negative BRCA (E) 
dysregulated IncRNAs were enriched in signal transduction, cell adhesion, cell cycle, mitosis, and cell division.

The estrogen receptor (ER) was overexpressed in nearly $70 \%$ of breast cancer cases [15]. The molecular subtypes of ER have been the main indicator of anti-hormonal therapy for breast cancer [26]. Moreover, ER-negative (ER-) BC exhibited dismal survival rates due to the highly aggressive and metastatic behavior. However, how ER leads to uncontrolled cell proliferation of breast cancer is yet to be fully understood [27, 28]. Moreover, few studies have focused on the dysregulation of IncRNAs in the ER-negative subtype breast cancer. In this study, by analyzing GSE70947, we found that C21orf81, PVT1, NBPF22P, PP14571, CYP4Z2P, FLJ40504, LOC440335, LOC145837, FLJ45983 were down-regulated and LOC375196 was up-regulated in ER-negative samples. Functional analysis of IncRNA-associated PPI modules in ER-negative breast cancer showed that these IncRNAs were associated with regulation of transcription, the response to estrogen stimulus, the tumor necrosis factor-mediated signaling pathway, negative regulation of cell growth, negative regulation of cell adhesion, interspecies interaction between organisms and cell-cell signaling.

Previous studies had shown that HER-2-positive breast cancer was more aggressive and had a shorter survival time [29]. In this study, by analyzing GSE70947, we found that FLJ45983, LOC440335, NEURL3, C9orf122, FLJ40504, LOC145837, PVT1, NCRNA00173, EGOT, and LOC283867 were down-regulated in HER2-positive samples. Functional analysis of IncRNA-associated PPI modules in HER2-positive breast cancer showed that these IncRNAs were associated with collagen catabolism, the estrogen receptor signaling pathway, glucose homeostasis, hormone metabolism, negative regulation of cell adhesion, positive regulation of neuron differentiation, the response to estrogen stimulus and regulation of cell proliferation.

Of note, analysis of the GSE70947 database showed that PVT1, LOC145837, FLJ40504 and FLJ45983 were significantly decreased in HER2-positive and ER-negative breast cancers. To explore the molecular mechanisms involved in these IncRNAs regulating HER2-positive and ER-negative breast cancer progression, we constructed IncRNA-miRNA-mRNA ceRNA networks based on our analysis. Our results showed that PVT1 played the most important roles. PVT1 was reported as an oncogene in different kinds of cancer, including non-small cell lung cancer [30] and breast cancer. However, whether PVT1 could act as a biomarker for breast cancer remains unknown. Here, we analyzed the Betastasis dataset and found that high PVT1 expression levels were associated with a lower survival rate in breast cancer patients.

\section{Conclusions}

We identified the significantly differentially expressed mRNAs and IncRNAs in breast cancer by using GSE70947. GO and KEGG pathway analysis showed that differentially expressed IncRNAs played key roles in regulating signal transduction, cell adhesion, the cell cycle, the insulin signaling pathway and the MAPK signaling pathway. The dysregulated IncRNA and mRNA expression profiles in HER2-positive and ER-negative breast cancer were also analyzed in this study. We also constructed the PVT1, LOC145837, FLJ40504 and FLJ45983 mediated ceRNA networks in HER2-positive and ER-negative breast cancers. Moreover, using the Betastasis dataset, we found that high PVT1 expression levels were associated with a lower survival rate in breast cancer patients. These results elucidate the functions of IncRNAs and provide useful information for exploring candidate therapeutic targets and new molecular biomarkers for ER-negative and HER-2 enriched subtype breast cancer.

\section{Acknowledgments}

We acknowledge excellent technical and graphic design assistance from Dr Jun Jiang.

\section{Conflict of interest}

The authors declare no conflict of interest.

\section{References}

1. Gupta GP, Massague J. Cancer metastasis: building a framework. Cell 2006; 127: 679-95.

2. Lin A, Li C, Xing Z, et al. The LINK-A IncRNA activates normoxic HIF1alpha signalling in triple-negative breast cancer. Nat Cell Biol 2016; 18: 213-24.

3. Prat A, Parker JS, Karginova O, et al. Phenotypic and molecular characterization of the claudin-low intrinsic subtype of breast cancer. Breast Cancer Res 2010; 12: R68.

4. Osborne CK. Steroid hormone receptors in breast cancer management. Breast Cancer Res Treat 1998; 51: 227-38.

5. Sorlie T, Perou CM, Tibshirani R, et al. Gene expression patterns of breast carcinomas distinguish tumor subclasses with clinical implications. Proc Natl Acad Sci U S A 2001; 98: 10869-74.

6. Finnegan TJ, Carey LA. Gene-expression analysis and the basal-like breast cancer subtype. Future Oncol 2007; 3: 55-63.

7. Mitri Z, Constantine T, O'Regan R. The HER2 receptor in breast cancer: pathophysiology, clinical use, and new advances in therapy. Chemother Res Pract 2012; 2012: 743193.

8. Sorlie T, Tibshirani R, Parker J, et al. Repeated observation of breast tumor subtypes in independent gene expression data sets. Proc Natl Acad Sci U S A 2003; 100: 8418-23. 
9. Chien AJ, Rugo HS. Emerging treatment options for the management of brain metastases in patients with HER2-positive metastatic breast cancer. Breast Cancer Res Treat 2013; 137: 1-12.

10. Amorim M, Salta S, Henrique R, Jerónimo C. Decoding the usefulness of non-coding RNAs as breast cancer markers. J Transl Med 2016; 14: 265.

11. Wan X, Huang W, Yang S, et al. Identification of androgen-responsive IncRNAs as diagnostic and prognostic markers for prostate cancer. Oncotarget 2016; 7 60503-18.

12. Kim T, Cui R, Jeon YJ, Fadda P, Alder H, Croce CM. MYC-repressed long noncoding RNAs antagonize MYC-induced cell proliferation and cell cycle progression. Oncotarget 2015; 6: 18780-9.

13. Geisler S, Coller J. RNA in unexpected places: long non-coding RNA functions in diverse cellular contexts. Nat Rev Mol Cell Biol 2013; 14: 699-712.

14. Huang NS, Chi YY, Xue JY, et al. Long non-coding RNA metastasis associated in lung adenocarcinoma transcript 1 (MALAT1) interacts with estrogen receptor and predicted poor survival in breast cancer. Oncotarget 2016; 7: 37957-65.

15. Niknafs YS, Han S, Ma T, et al. The IncRNA landscape of breast cancer reveals a role for DSCAM-AS1 in breast cancer progression. Nat Commun 2016; 7: 12791.

16. Zhang X, Sun S, Pu JK, et al. Long non-coding RNA expression profiles predict clinical phenotypes in glioma. Neurobiol Dis 2012; 48: 1-8.

17. Franceschini A, Szklarczyk D, Frankild S, et al. STRING v9.1: Protein-protein interaction networks, with increased coverage and integration. Nucleic Acids Res 41 (D1):

18. Saito R, Smoot ME, Ono K, et al. A travel guide to Cytoscape plugins. Nat Methods 2012; 9: 1069-76.

19. Adamcsek B, Palla G, Farkas IJ, Derényi I, Vicsek T. CFinder: Locating cliques and overlapping modules in biological networks. Bioinformatics 2006; 22: 1021-3.

20. Guttman M, Rinn JL. Modular regulatory principles of large non-coding RNAs. Nature 2012; 482: 339-46.

21. Shen X, Xie B, Ma Z, et al. Identification of novel long non-coding RNAs in triple-negative breast cancer. Oncotarget 2015; 6: 21730-9.

22. Lewis BP, Burge CB, Bartel DP. Conserved seed pairing often flanked by adenosines, indicates that thousands of human genes are microRNA targets. Cell 2005; 120: 15-20.

23. Guan Y, Kuo WL, Stilwell JL, et al. Amplification of PVT1 contributes to the pathophysiology of ovarian and breast cancer. Clin Cancer Res 2007; 13: 5745-55.

24. Siegel R, Naishadham D, Jemal A. Cancer statistics. CA Cancer J Clin 2012; 65: 10-29.

25. Wang X, Sehgal L, Jain N, Khashab T, Mathur R, Samaniego F. LncRNA MALAT1 promotes development of mantle cell lymphoma by associating with EZH2. J Transl Med 2016; 14: 346.

26. Narayanan R, Dalton JT. Androgen receptor: a complex therapeutic target for breast cancer. Cancers (Basel) 2016; 8: 108.

27. Kümler I, Knoop AS, Jessing CAR, Ejlertsen B, Nielsen $D L$. Review of hormone-based treatments in postmenopausal patients with advanced breast cancer focusing on aromatase inhibitors and fulvestrant. ESMO Open 2016; 1: e000062.

28. Lousberg L, Collignon J, Jerusalem G. Resistance to therapy in estrogen receptor positive and human epidermal growth factor 2 positive breast cancers: progress with latest therapeutic strategies. Ther Adv Med Oncol 2016; 8: 429-49.

29. Kelly CM, Janjigian YY. The genomics and therapeutics of HER2-positive gastric cancer-from trastuzumab and beyond. J Gastrointest Oncol 2016; 7: 750-62.

30. Liu FT, Xue QZ, Zhu ZM, et al. Long noncoding RNA PVT1, a novel promising biomarker to predict lymph node metastasis and prognosis: a meta-analysis. Panminerva Med 2016; 58: 160-6. 